\title{
Sudden Cardiac Death in Patients Undergoing Dialysis: \\ More than a single toxin
}

\author{
Sharon M. Moe, MD \\ Division of Nephrology, Indiana University School of Medicine \\ 950 W. Walnut Street, R2-202 \\ Indianapolis IN 46202 \\ Phone: 3172782868
}

Fax: 317 274-8575

Word count: 964 including references

No conflicts of interest or acknowledgements.

This is the author's manuscript of the article published in final edited form as:

Moe, S. M. (2018). Sudden Cardiac Death in Patients Undergoing Dialysis: More than a single toxin. Heart Rhythm. https://doi.org/10.1016/j.hrthm.2018.09.017 
Dialysis is a lifesaving procedure, or perhaps life prolonging is more appropriate. The native kidneys work 24 hours a day, 7 days a week and filter over 1000 Liters of blood per week. In addition, to concentrating the filtrate (urine), the kidney tubules reabsorb, metabolize, and secrete various metabolites. Thus, kidney failure results in the accumulation of fluid, and accumulation of multiple toxins leading to systemic symptom. Unfortunately, dialysis does not correct the majority of these abnormalities. Typical hemodialysis done thrice weekly only 'filters' one tenth of the normal kidney, only clears toxins less than 15,000 Daltons, and does not metabolize or synthesize. In other words, it is not a kidney replacement. The result is systemic derangements in multiple metabolic pathways commonly referred to as a "uremic milieu"- an environment that is detrimental to normal physiologic processes.

With the initiation of dialysis, some of these abnormalities are corrected, most notably volume overload, but most are not corrected, and many other abnormalities are created. As noted in this issue by Tereshchenko et $\mathrm{al}^{1}$, the first few months of dialysis are associated with very high mortality; the etiology of which has been thought secondary to the sudden shifts of electrolytes and volume in individuals with likely small vessel atherosclerosis or arteriosclerosis. Once patients survive the first few months of undergoing dialysis, the leading cause of death is sudden cardiac death (SCD)- responsible for 25-35\% of all cardiovascular deaths in studies with adjudicated endpoints ${ }^{2}$. However, the risk of sudden cardiac death increases with progressive kidney disease even prior to the onset of dialysis such that the procedure of dialysis cannot be completely to blame ${ }^{3}$. Furthermore, the cause of SCD differs from that observed in the general population where ventricular rhythms are most commonly observed. A recent study evaluated arrhythmias in 44 patients undergoing hemodialysis through implantable loop recorders with continuous cardiac rhythm monitoring. The most common arrhythmia was bradycardia with 14 episode of asystole, and only one sustained ventricular tachycardia ${ }^{4}$, results confirmed in our animal study ${ }^{5}$. Thus, patients undergoing dialysis have a unique physiology of SCD that deserves further explanation.

Tereshechenko ${ }^{1}$ argues that toxins released from dialyzers, plastic tubing, and dialysis catheters, including polyvinyl chloride (PVC), bisphenol A (BPA), and Di(2-ethylhexyl) phthalate (DEHP) may be a pathogenic cause of sudden cardiac death. The concept that the dialyzer may be contributing to poor outcomes is not new. The majority of dialyzers used today are made of synthetic polymer blends. The use of synthetic dialyzers began in the 1980s after recognition that cellulose based membranes induced acute inflammation ${ }^{6}$. Bosch-Panadero demonstrated in 2016 that bisphenol A (BPA) is indeed released from these 'new' synthetic membranes and can also induce inflammation and oxidative stress as did the older cellulose based membranes ${ }^{7}$. Tereschechenko ${ }^{1}$ puts forth the hypothesis of a link between the toxins released from plastics and SCD based on in vitro studies demonstrating that DEHP impairs electrical conduction in cardiomyocytes. They further note that hemodialysis, compared to peritoneal dialysis, is associated with an increased risk of SCD and argue that the increased plastic exposure with hemodialysis may explain the difference in risk. However, patients receiving peritoneal dialysis, compared to hemodialysis, also have preserved residual renal function secondary to the more gentle fluid removal and less hypotension with peritoneal dialysis. This preserved residual renal function is associated with preserved metabolic function of the kidney and one study even showed improved left 
ventricular structure ${ }^{8}$. Further, no studies to date have associated levels of these toxins with mortality or cardiovascular death in dialysis patients. Thus, the proposed arguments supporting a causative role of the plastic metabolites have significant limitations, especially when one considers the uremic milieu and the large number of other uremic toxins that have been associated with cardiovascular death. However, the lack of convincing data does not negate the need to think twice about adding iatrogenic insults to patients with multiple and significant risk factors for SCD. Unfortunately, we have yet to develop a better alternative to dialysis (other than transplant). Nevertheless, we should at least strive to make the procedure as safe as possible.

\section{REFERENCES:}

1. Tereshchenko, LG, Posnack, NG: Does plastic chemical exposure contribute to sudden death of patients on dialysis? Heart Rhythm, 2018.

2. Wheeler, DC, London, GM, Parfrey, PS, Block, GA, Correa-Rotter, R, Dehmel, B, Drueke, TB, Floege, J, Kubo, Y, Mahaffey, KW, Goodman, WG, Moe, SM, Trotman, ML, Abdalla, S, Chertow, GM, Herzog, CA, Investigators, EVOCHTtLCET: Effects of cinacalcet on atherosclerotic and nonatherosclerotic cardiovascular events in patients receiving hemodialysis: the EValuation of Cinacalcet $\mathrm{HCl}$ Therapy to Lower CardioVascular Events (EVOLVE) trial. J Am Heart Assoc, 3: e001363, 2014.

3. Green, D, Roberts, PR, New, DI, Kalra, PA: Sudden cardiac death in hemodialysis patients: an in-depth review. Am J Kidney Dis, 57: 921-929, 2011.

4. Roy-Chaudhury, P, Tumlin, JA, Koplan, BA, Costea, Al, Kher, V, Williamson, D, Pokhariyal, S, Charytan, $\mathrm{DM}, \mathrm{Mi}, \mathrm{Di}$, committees: Primary outcomes of the Monitoring in Dialysis Study indicate that clinically significant arrhythmias are common in hemodialysis patients and related to dialytic cycle. Kidney Int, 93: 941-951, 2018.

5. Hsueh, CH, Chen, NX, Lin, SF, Chen, PS, Gattone, VH, 2nd, Allen, MR, Fishbein, MC, Moe, SM: Pathogenesis of Arrhythmias in a Model of CKD. J Am Soc Nephrol, 2014.

6. Keshaviah, PR, Luehmann, DA: Risks and hazards associated with dialyzers and dialysate delivery systems. Crit Rev Biomed Eng, 9: 201-244, 1983.

7. Bosch-Panadero, E, Mas, S, Sanchez-Ospina, D, Camarero, V, Perez-Gomez, MV, Saez-Calero, I, Abaigar, P, Ortiz, A, Egido, J, Gonzalez-Parra, E: The Choice of Hemodialysis Membrane Affects Bisphenol A Levels in Blood. J Am Soc Nephrol, 27: 1566-1574, 2016.

8. Rebic, D, Matovinovic, MS, Rasic, S, Kes, P, Hamzic-Mehmedbasic, A: The Effect of Preserved Residual Renal Function on Left Ventricular Structure in Non-Anuric Peritoneal Dialysis Patients. Kidney Blood Press Res, 40: 500-508, 2015. 